\title{
Solidification and ordering during directional drying of a colloidal dispersion
}

\author{
Lucas Goehring, ${ }^{1,2,3}$ William J. Clegg, ${ }^{1}$ and Alexander F. Routh ${ }^{2,3, *}$ \\ ${ }^{1}$ Department of Materials Science and Metallurgy, \\ University of Cambridge, Pembroke Street, Cambridge, UK, CB2 3QZ \\ ${ }^{2}$ Department of Chemical Engineering and Biotechnology, \\ University of Cambridge, Pembroke Street, Cambridge, UK, CB2 3RA \\ ${ }^{3}$ BP Institute for Multiphase Flow, University of Cambridge, \\ Madingley Rise, Madingley Road Cambridge, UK, CB3 OEZ
}

(Dated: March 12, 2010)

\begin{abstract}
During drying, colloidal dispersions undergo processes such as solidification, cracking, and the draining of interstitial pores. Here we show that the solidification of polystyrene and silica dispersions, during directional drying, occurs in two separate stages. These correspond to the initial ordering, and subsequent aggregation, of the colloidal particles. Transitions between these stages are observed as changes in transparency and color that propagate as distinct fronts along the drying layer. The dynamics of these fronts are shown to arise from a balance between compressive capillary forces, and the electrostatic and van der Waals forces described by DLVO theory. This suggests a simple method by which the maximum inter-particle repulsion between particles can be measured through the optical inspection of the dynamics of a drying dispersion, under a microscope.
\end{abstract}

\section{INTRODUCTION}

Colloidal dispersions, such as those used in the production of paints, ceramics, or photonic crystals, typically dry directionally $[1,2]$. During directional drying a film dries first at its edge, leaving a slowly shrinking liquid area. A series of drying fronts appear, as shown in 1, and propagate into the still-liquid dispersion. The appearance of three distinct fronts have frequently been reported, which represent solidification, cracking, and pore opening (e.g. [3-8]). For films that are dried below their critical film thickness, the fracture front is absent, and the film dries without breaking [3]. These processes have been shown to be driven by the compressive capillary forces that act on the menisci between drying colloidal particles [3-8].

When compressed, the particles of a colloidal dispersion irreversibly aggregate when the pressure reaches some critical aggregation pressure. This was originally demonstrated through a series of centrifuge experiments, which showed that this critical pressure corresponded to the maximum pressure that could be generated by the repulsive interactions between adjacent colloidal particles [9-11]. The connection of aggregation to drying was then made by Chiu et al., who argued that drying colloidal alumina will solidify only after the repulsive interactions between particles were overcome by capillary forces [3], and by Dufresne et al., who also noted the presence of such an aggregation front in drying ludox [6]. The work of Tsapis et al. has further demonstrated the existence of a fluid-to-solid transition in evaporating colloidal droplets, at a fixed aggregation pressure, when the capillary forces balanced the electrostatic repulsion of adjacent particles [12].

*afr10@cam.ac.uk

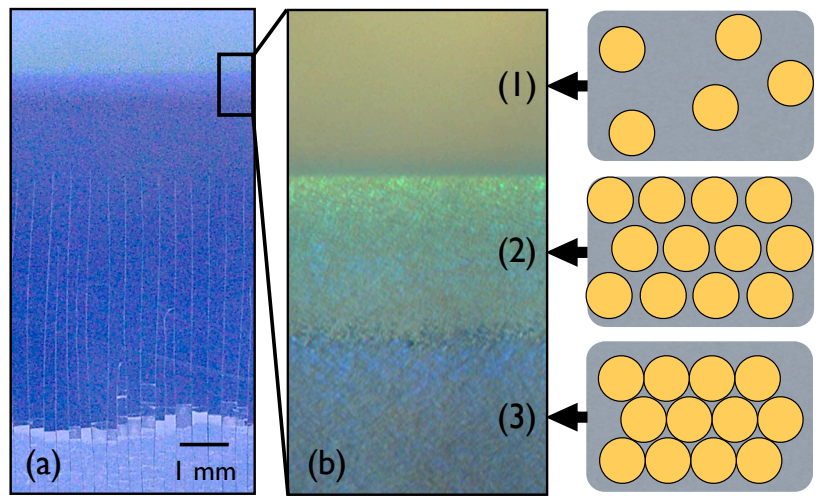

FIG. 1: When a colloidal suspension dries directionally, a series of fronts advance into a shrinking liquid pool. (a) In a dispersion of $115 \mathrm{~nm}$ polystyrene particles, still liquid at the top of this image, these fronts appear as changes in transparency, or the appearance of a parallel array of cracks. (b) When $198 \mathrm{~nm}$ diameter particles are used, the change from liquid dispersion to solid film is highlighted by opalescent color. In the liquid region (1), the particles are randomly dispersed in liquid. In the transition region (2), the appearance of color indicates an ordered structure. In passing to the final region (3), the change in color indicates a change in structure, which is interpreted as the irreversible aggregation of the particles.

Here we consider, in detail, how a colloidal dispersion changes from a liquid to solid when drying, and show that the process of solidification happens in two separate stages. We interpret these stages as the solidification of colloidal particles into a non-touching network, followed by the collapse of this network when the particles irreversibly aggregate. Both effects are argued to proceed from the interaction of capillary pressure with the inter-particle forces of colloidal dispersions described by DLVO theory. By exploiting these effects, we demonstrate a simple method whereby the maximum repulsive force felt between colloidal particles can be measured by 
observing the position and velocity of drying fronts in a thin colloidal film, under a microscope.

This study uses the phenomenon of structural color, or opalescence, in colloidal dispersions to infer properties of those dispersions. These colors occur when the arrangement of the colloidal particles preferentially scatters light of certain wavelengths. The shifting colors of light, when opals are viewed from different angles, represent Bragg diffraction patterns with respect to the visible spectrum, caused by the crystalline arrangement of particles $[13,14]$. Alternatively, the arrangement of particles in a glassy, or amorphous state, possesses high translational order, but not orientational order [15]. In a glass, the well-defined average inter-particle separation can lead to structural color without a strong angular variation of wavelength [16, 17]. Structural colors can be difficult to interpret quantitatively, but the sudden appearance, or change, in color indicates a change in the structure of the colloidal particles, or in inter-particle spacing.

\section{MATERIALS AND METHODS}

Polystyrene dispersions were synthesized by emulsion polymerization, using a recipe adapted from Ottewill and Shaw [18], with $\mathrm{K}_{2} \mathrm{~S}_{2} \mathrm{O}_{8}$ as the initiator, $\mathrm{NaHCO}_{3}$ as the buffer, and sodium dodecyl sulphate as the surfactant. A multi-stage growth was performed, where the product of each stage was used as a seed for subsequent growth. Dispersions with particle diameters of 115, 144, and $198 \mathrm{~nm}$, and poly-dispersities of up to $15 \%$, were produced. The solid contents of the dispersions were $9-12 \%$, by volume, after extensive dialysis. Size distributions were obtained using dynamic light scattering (Brookhaven ZetaPALS). The particle surface charge of the $198 \mathrm{~nm}$ polystyrene dispersion was measured by potentiometric titration to be $0.53 \pm 0.05 \mu \mathrm{C} / \mathrm{cm}^{2}$, in good agreement with similarly prepared colloids $[11,19]$. Dispersions of colloidal silica (Ludox AM), with a measured average particle size of 38 $\mathrm{nm}$, were used for additional observations. All chemicals were obtained from Sigma-Aldrich, and certified $\geq 99 \%$ pure, and deionized water (Elgastat Option 3) was used at all times.

Solutions of known $\mathrm{KCl}$ concentrations, from 0-150 $\mathrm{mM}$, were prepared in a volumetric flask. Mixtures of one part of saline solution to four parts dispersion were made. An initial ionic concentration of $0.4 \mathrm{mM}$ was assumed to be present in the dialyzed dispersions, as estimated from conductivity measurements. This background concentration is largely due to the counterions associated with the particle surface charges.

Liquid films of the dispersions were spread flat onto level $50 \times 75 \mathrm{~mm}^{2}$ glass microscope slides and allowed to dry by evaporation from their exposed upper surface. Under these conditions the films dried directionally, with solidification starting along all film edges, and proceeding inwards toward the film centre. Films were dried at room temperature, with no additional control over drying

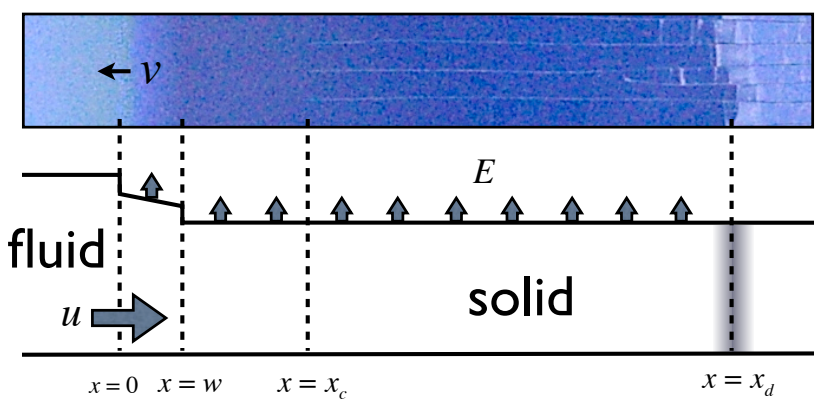

FIG. 2: Four distinct fronts can be visible in directionally dried dispersions: an ordering front at $x=0$, an aggregation front at $x=w$, a fracture front at $x=x_{c}$, and a poreemptying front at $x=x_{d}$. The fronts move at an average velocity $v$ into the liquid region, caused by a superficial fluid velocity $u$ into the solidified region. Evaporation, at a rate $E$ per unit area, over the saturated film balances the flow into this region.

conditions. A digital microscope was used to collect timelapse images every few seconds, from which the positions and velocities of drying features were extracted. The 198 $\mathrm{nm}$ polystyrene particles displayed structural color during drying, with predominantly green-purple colors. A number of films were cast for each dispersion, with different film thicknesses and salt concentrations. When dry, these were typically in the range of 10 to $100 \mu \mathrm{m}$ thick.

\section{RESULTS}

The solidification of polystyrene and silica dispersions, during directional drying, was observed to occur in two separate stages. As drying proceeds, these two stages are separated in space, and form two fronts that propagate along the drying dispersion, as shown in 1 . The geometry of the drying film is sketched in 2 . The leading solidification, or ordering, front is taken as $x=0$, and the trailing solidification, or aggregation, front is at $x=w$. Unless otherwise stated, observations were repeated across all polystyrene, and silica dispersions.

When dispersions of $198 \mathrm{~nm}$ polystyrene are dried, and observed under an optical microscope, the leading solidification front is associated with the appearance of bright color. This opalescence is suggestive of a crystalline array of particles, which diffracts visible light [20]. This interpretation was confirmed through retrospective atomic force microscopy. As shown in 3, the individual polystyrene particles are arranged into a polycrystalline structure in the dried film.

There are gradual changes in the hue of the film between the two solidification fronts, and a sharp change in color at the trailing front, as shown in 1(b). The shift in the dominant color from green to blue represents a decrease in the wavelength of the diffracted light, and suggests that the inter-particle spacing is also decreas- 


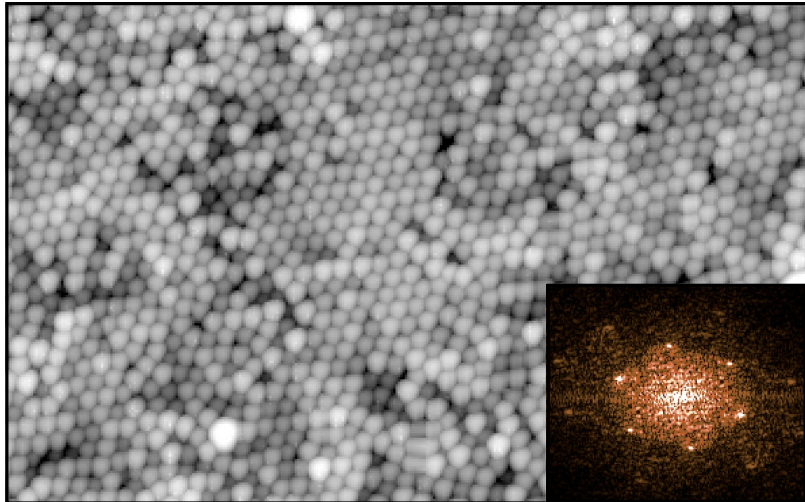

FIG. 3: Atomic force micrograph showing polycrystalline structure at the surface of a dried film containing $198 \mathrm{~nm}$ polystyrene particles. The image shows the relative surface height over a $5 \times 8 \mu \mathrm{m}^{2}$ region. The inset shows the $2 \mathrm{D}$ Fourier transform of the surface height profile, with spots indicating a hexagonally packed crystalline arrangement.

ing. This was tested by panning the focal plane of the microscope, and the color changes were seen to correspond to appropriate changes in the height of the film. In dispersions of $115 \mathrm{~nm}$ or $144 \mathrm{~nm}$ polystyrene, or silica, the two solidification fronts appear as changes in the transparency of the film, as shown in 1(a). Atomic force microscopy showed that the $144 \mathrm{~nm}$ polystyrene particles did crystallize, but that the $115 \mathrm{~nm}$ polystyrene particles formed into an amorphous, glassy structure. We would tentatively associate the onset of this glassy state with the change in transparency at what we term the ordering front.

If a partially dried film is gently flooded with water, the region between the two fronts re-disperses, whilst the material behind the trailing front, $x \geq w$, remains solid, as shown in 4 . This demonstrates that the first, ordering, transition is reversible, but the second front represents an irreversible change in the nature of the dispersion.

A micro-manipulator was used to probe the surface of a drying film with a fine drawn-glass tip. The liquid dispersion forms a temporary meniscus around the tip, when touched. For $x>w$ the solid film can be scratched or broken, depending on the force applied by the tip. If the tip is brought into contact with the surface between the two drying fronts, $0<x<w$, a meniscus forms, but the material in this region does not return to its original shape when the tip is removed. Instead, a permanent peak is left at the point where the tip is withdrawn. This demonstrates that the region between the two solidification fronts is capable of sustaining a rigid deformation.

The two solidification fronts show different dynamics, but are intimately coupled to each other. Their behavior was observed in dispersions of $198 \mathrm{~nm}$ polystyrene, at concentrations of $\mathrm{KCl}$ up to $14 \mathrm{mM}$. In a small number of cases, both fronts advanced smoothly, maintaining a constant speed $v$ and separation $w$. More frequently the fronts displayed an intermittent, or 'stick-slip' motion.

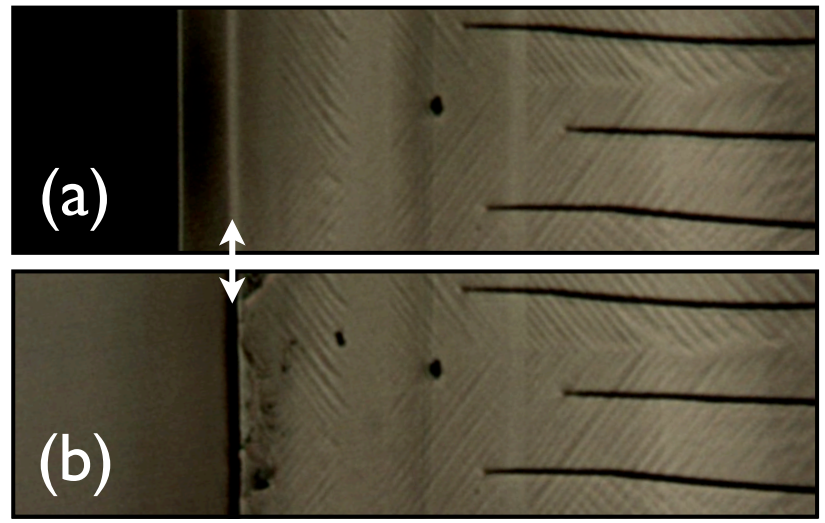

FIG. 4: If a drying colloidal dispersion containing $115 \mathrm{~nm}$ diameter polystyrene particles (a) is gently flooded with water, then (b) the region between the two solidification fronts redisperses, but the region behind the trailing front (marked by white arrows) remains solid. The time between images is $20 \mathrm{~s}$.

In these cases, the trailing solidification front is retarded, or even stationary, while the leading front advances. The leading front slows down as the separation $w$ increases. Eventually the aggregation front quickly advances, the front separation decreases, and the ordering front speeds up. This cycle, which typically lasts a few tens of seconds, then repeats, indefinitely. The aggregation front leaves visible marks behind when it advances, which can be seen by the naked eye as a series of concentric rings on the final dried film. This intermittent behavior was observed at all salt concentrations that were prepared. Demonstrations are shown in $14 \mathrm{mM} \mathrm{KCl}$, in $5(\mathrm{a})$, and in $0.6 \mathrm{mM} \mathrm{KCl}$, in the online supplemental movie 1.

The intermittent advances of the aggregation front appear to be correlated with a similar behavior of the fracture front. This was directly observed in colloidal silica, in a suspension with $30 \mathrm{mM} \mathrm{KCl}$, where the solidification and fracture fronts are compact enough to be observed simultaneously under high optical magnification. When the aggregation front speeds up the cracks can be seen to all jump forward simultaneously. This is shown in 5(b), and the online supplemental movie 2. The advances of the aggregation front are not caused by jumps in the fracture front, as the intermittent behavior of the solidification fronts is also seen very early into drying, before any cracks have formed. These observations suggest that either the advance of the solidification front causes the cracks to advance, or that both are triggered by some additional factor.

The separation, $w$, between the ordering and aggregation fronts is inversely correlated with the speed $v$ of the leading front. This correlation holds both instantaneously, as is suggested by the data in 5(a), and as an average behavior. $198 \mathrm{~nm}$ colloidal polystyrene was dried at various $\mathrm{KCl}$ concentrations. At each concentration and film thickness, the width $w$ and speed $v$ were measured every few seconds. The inverse relationship be- 

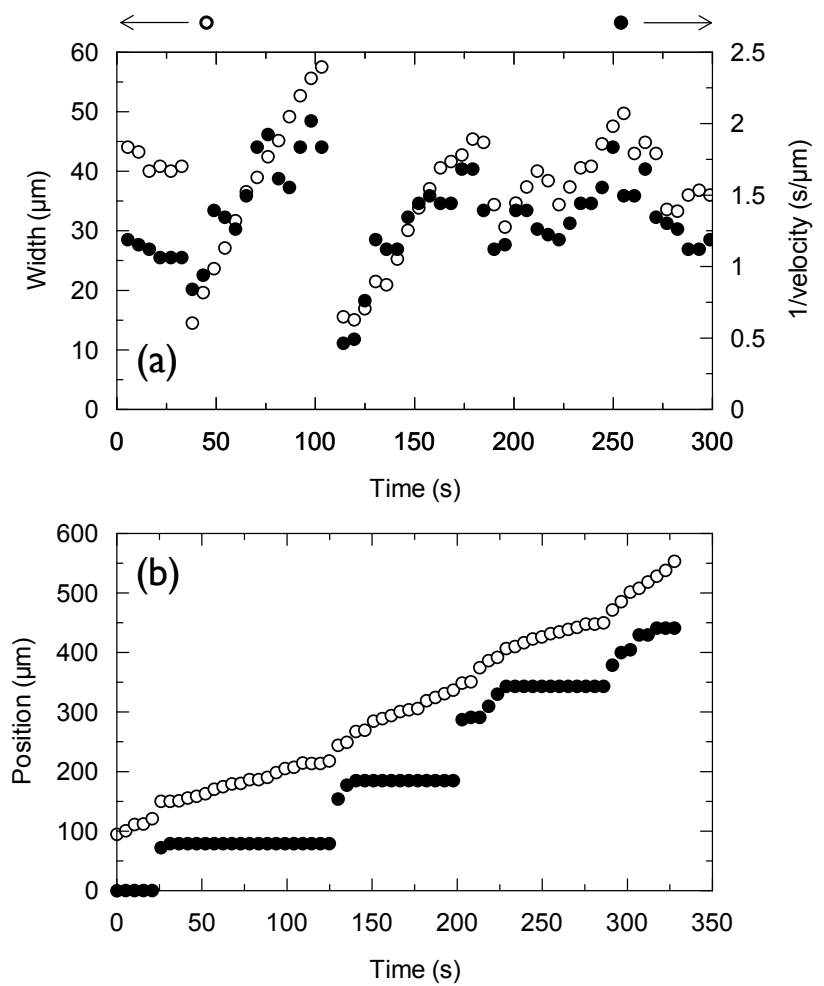

FIG. 5: (a) The two solidification fronts do not always advance smoothly into the drying dispersion. As the distance between the fronts, $w$ increases (open circles), the velocity, $v$, of the leading front slows down, to maintain an inverse relationship between $v$ and $w$ (closed circles show $1 / v$ ). (b) In drying colloidal silica (ludox), each time the position of the aggregation front (open circles) accelerates, the fracture front (solid circles) also advances, as many cracks simultaneously advance.

tween these variables is shown in 6(a), and includes data for all film thicknesses tested. The data were averaged over 5 measurements to reduce experimental noise, and the reported salt concentrations include an initial ionic concentration of $0.4 \mathrm{mM}$, in the dialyzed dispersion. For each salt concentration, the error-weighted mean value of the product $v w$ was found. As shown in 6(b), the addition of salt decreases this product.

\section{THEORY}

The two-stage process of solidification of drying colloidal dispersions can be understood in terms of the interactions between colloidal particles. As evaporation occurs, the colloidal dispersion becomes more concentrated, and thermodynamic considerations drive an ordering of the particles into a crystalline network [20], or glassy state $[16,20]$. Fluid flow through this porous network leads to a pressure drop that compresses the particles. When the pressure exceeds the maximum repulsive pressure that can be supplied by the inter-particle potentials,
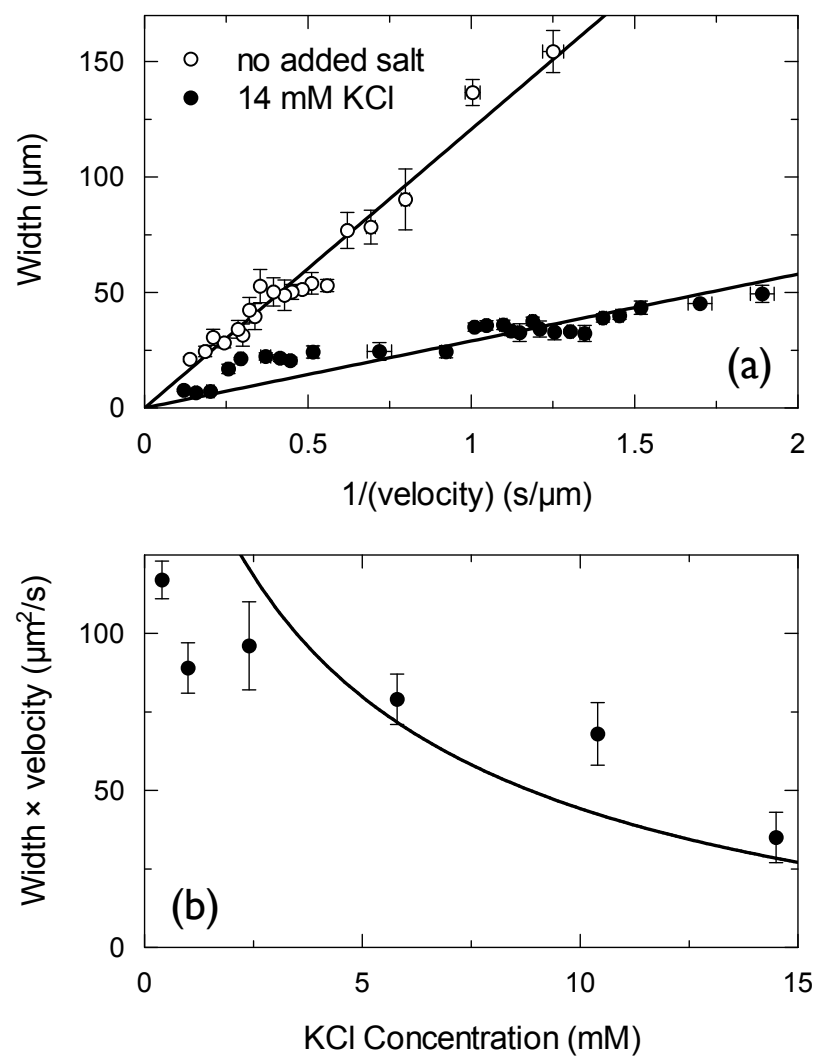

FIG. 6: (a) The separation $w$ of the ordering and aggregation fronts scales inversely with the velocity $v$ of the initial, ordering front. $198 \mathrm{~nm}$ polystyrene spheres are used, with an $7.5 \%$ initial solids volume fraction. Data are averaged over 5 measurements of $w$ and $v$, to reduce experimental noise. (b) As salt is added to the system, the fronts become closer together. The solid line is the prediction of Eqn. 11.

the particles irreversibly aggregate.

In the Derjaguin-Landau-Verway-Overbeek (DLVO) theory of colloidal interactions, the force, $F$, between two identical spherical particles of radius $a$ and surface separation $s$ is the sum of their electrostatic and van der Waals forces,

$$
F=-\frac{A a}{12 s^{2}}+32 \pi \epsilon a \kappa\left(\frac{k T}{e}\right)^{2} \tanh ^{2}\left(\frac{e \Phi}{4 k T}\right) e^{-\kappa s} .
$$

Here $A$ is the Hamaker constant, taken to be $5 \times 10^{-21}$ $\mathrm{J}$ for polystyrene in water [21], $\epsilon$ is the permittivity of the liquid dispersant, $e$ is the charge of an electron, $k$ is Boltzmann's constant, and $T=293 \mathrm{~K}$ is the temperature. The electrostatic interaction is screened by ions in solution, and therefore takes an exponential form, decaying with Debye length $\kappa^{-1}=(0.304 \mathrm{~nm}) M^{-1 / 2}$, in a $\mathrm{KCl}$ solution of molarity $M[21]$. The surface potential $\Phi$ is calculated using the measured surface charge density $\sigma=0.53 \mu \mathrm{C} / \mathrm{cm}^{2}$, and the approximation [22]

$$
\sigma=\frac{\epsilon \kappa k T}{e}\left(2 \sinh \left(\frac{e \Phi}{2 k T}\right)+\frac{4}{\kappa a} \tanh \left(\frac{e \Phi}{4 k T}\right)\right) .
$$


The forces of Eqn. 1 are repulsive at long range, but become attractive at particle separations of a few nm, as shown in 7 .

The ordering of a dispersion of mono-disperse spheres into a non-touching crystalline array is known to occur at an effective hard sphere volume fraction of about 0.52 [20]. Electrostatic repulsion increases the effective diameter of particles such that the fluid-solid transition occurs at lower particle volume fractions [20]. For a $\mathrm{KCl}$ concentration of $1 \mathrm{mM}$, the ordering of $100 \mathrm{~nm}$ radius particles should occur at a volume fraction of about 0.39 , or an inter-particle separation of $24 \mathrm{~nm}$.

In a drying colloidal system, liquid flows through the saturated solid film, and is ultimately lost by evaporation. This can be considered as a 1D system, as sketched in 2. The superficial fluid velocity $u$ is maintained by a gradient in the pressure $p$, as described by Darcy's law,

$$
\frac{\partial p}{\partial x}=-\frac{\mu}{\kappa_{p}} u
$$

where $\mu$ is the viscosity of the fluid, and $\kappa_{p}$ is the permeability of the network, frequently approximated by the Carmen-Kozeny equation [5, 23],

$$
\kappa_{p}=a^{2}(1-\phi)^{3} / 45 \phi^{2} .
$$

Over the upper surface of the film evaporation proceeds at a constant rate $E$. Given a film of height $h$ conservation of fluid implies that $\partial u / \partial x=-E / h$. By differentiating Eqn. 3 one then obtains

$$
\frac{\partial^{2} p}{\partial x^{2}}=\frac{\mu E}{\kappa_{p} h},
$$

which has been used to describe the gradients of capillary pressure in drying porous films [2, 24].

Within the liquid region, pressure is due to surface tension, and the magnitude of this pressure is much lower than that generated by flow through the solid region [2]. Hence a boundary condition $p(0)=0$ is appropriate. Conservation of volume at the fluid-solid transition provides a second boundary condition

$$
u(0)=v \frac{\phi-\phi_{0}}{\phi_{0}}
$$

where $v$ is the solidification front speed, and $\phi_{0}$ is the solids fraction of the still fluid dispersion. The solution to Eqn. 5 with these boundary conditions is

$$
p(x)=-\frac{\mu}{\kappa_{p}}\left[v x \frac{\phi-\phi_{0}}{\phi_{0}}-\frac{E x^{2}}{2 h}\right] .
$$

In order to determine the capillary pressure at the aggregation front, $p(w)$, we simplify Eqn. 7 by linearizing

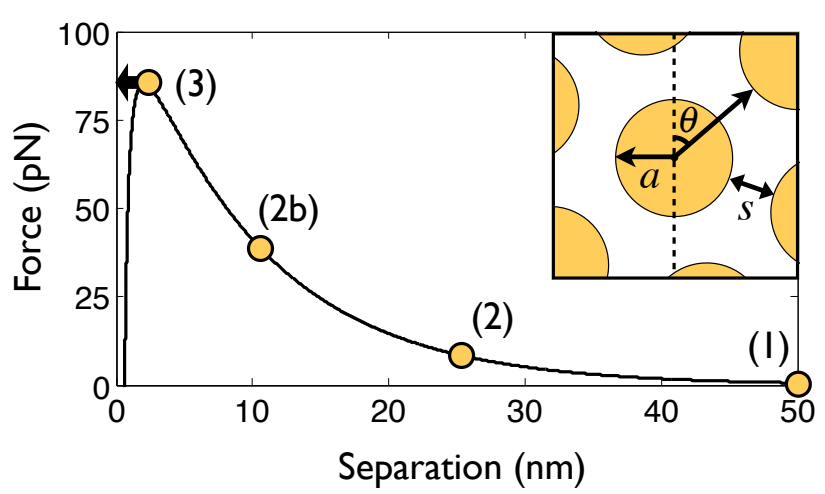

FIG. 7: The DLVO prediction, given by Eqn. 1, for the interparticle force between two particles is shown here for $198 \mathrm{~nm}$ polystyrene particles in a $1 \mathrm{mM} \mathrm{KCl}$ solution. At (1) low solids volume fraction, $\phi$, the particles are dispersed, and unordered. When $\phi$ is sufficiently high (2), the particles will self-assemble into a non-touching network. The further addition of compressive capillary forces causes $(2 \mathrm{~b})$ a decrease in the inter-particle spacing. When (3) the capillary force balances the maximum inter-particle repulsion, the particles jump into intimate contact, and irreversibly aggregate. We argue that the regions delimited by (1)-(3) correspond to the regions shown optically in $1(\mathrm{~b})$. The inset demonstrates the particle radius $a$, inter-particle separation, $s$, and angle between particles, $\theta$.

around $x=0$, and find

$$
p(w) \approx-v w \frac{\mu\left(\phi-\phi_{0}\right)}{\kappa_{p} \phi_{0}} .
$$

This approximation is valid when $u(0) \gg E w / 2 h$, which requires that the evaporation occurring between $x=0$ and $x=w$ is small compared to the evaporation over the entire solidified region. This will be the case if $w$ is small compared to $x_{d}$, the distance between the solidification and pore-opening fronts. In the experiments reported here, $x_{d}$ is typically $5-10 \mathrm{~mm}$, whilst $w$ is less than $150 \mu \mathrm{m}$, justifying this assumption. Eqn. 8 predicts that if the pressure that causes an irreversible collapse of the particle network is a constant, for a given salt concentration, then the product $v w$ will also be constant, as was demonstrated in 6 . For the data shown in 6(a), and taking $\phi=0.5$ as a representative value for the nontouching solid network, Eqn. 8 estimates that the critical aggregation pressure $p(w)$ is $-6 \mathrm{kPa}$ and $-2 \mathrm{kPa}$, for salt concentrations of 0.4 and $14 \mathrm{mM}$, respectively.

The effect of capillary pressure is to compresses the particle network. A force $\pi a^{2} p$ will push down on each particle, and this force will be distributed across the $n$ neighbors pushing down on that particle from above. If a contact is at an angle $\theta$ to vertical (see inset to 7 ), then the vertical force due to that contact is $F_{p} \cos \theta$, where $F_{p}$ is the compressive force countered by the inter-particle potential. Averaging over all possible arrangements on 
the upper half-sphere provides

$$
\pi a^{2} p=\frac{n}{2 \pi} \int_{0}^{2 \pi} \int_{0}^{\pi / 2} F_{p} \cos \theta \sin \theta d \theta d \phi^{\prime}=\frac{n F_{p}}{2} .
$$

In a close-packed crystalline arrangement, there will be twelve nearest neighbors, with $n=6$ above the horizontal plane through the particle. Therefore

$$
F_{p} \simeq \frac{\pi a^{2} p}{3}
$$

In a glassy arrangement, $n<6$, and will depend on the structure of the network.

As the particles are pushed together, their equilibrium separation changes, as sketched in 7 . This would cause a decrease in the dominant wavelength of the diffracted light, and a decrease in the height of the film, as observed. When the magnitude of the inter-particle compression $F_{p}$ reaches the maximum in the DLVO force-distance curve, $F_{\max }$, electrostatic repulsion will no longer be able to hold the particles apart, the particles will jump into intimate contact, and the particle network will irreversibly collapse. This effect is seen at the aggregation front, which marks the position at which the drying film cannot be easily re-dispersed in water. By combining the condition $F_{p}=-F_{\max }$ with Eqns. 4,8 and 10 , one obtains

$$
F_{\text {max }}=\mu v w \frac{15 \pi \phi^{2}\left(\phi-\phi_{0}\right)}{\phi_{0}(1-\phi)^{3}}
$$

As salt is added to the system, the magnitude of $F_{\max }$ decreases, and the product of $v$ and $w$ will accordingly decrease. The predicted behavior for $v w$ is shown in 6(b), taking $\phi=0.5$, and this prediction is in reasonable agreement with both the magnitude and trend of the experimental observations.

\section{SIMPLIFIED MODEL}

The scaling behavior of the solidification region can be highlighted by noting that the superficial fluid velocity, $v$, the evaporation rate $E$, and the position of the poredraining front, $x_{d}$, are all related. Here we assume that all fronts are propagating at a fixed velocity $v$, such that $w$ and $x_{d}$ are constants, averaged over time.

An overall water mass balance over the saturated film equates

$$
v \frac{\phi-\phi_{0}}{\phi_{0}}=\frac{x_{d} E}{h}
$$

At the pore-draining front, the capillary pressure reaches some maximum $p_{\max }$. This pressure is related to the minimum radius of curvature of the water-air interfaces that can be supported by the inter-particle pores, and has the form $p_{\max }=C \gamma / a$, where $\gamma$ is the surface tension of water, and $C$ is a geometry-dependant factor of order one [25]. By evaluating Eqn. 7 at $x_{d}$, and simplifying using Eqn. 12, one also finds

$$
x_{d}=\left(\frac{2 h \kappa_{p}}{\mu E} p_{\max }\right)^{1 / 2} .
$$

The condition expressed by Eqn. 11 can therefore be expressed solely in terms of process variables, and the observable $w$, as

$$
F_{\max }=\pi w\left(\frac{2 C a^{3} E \gamma \mu}{9 \kappa_{p} h}\right)^{1 / 2} .
$$

An alternative, but analogous approach is to simplify Eqn. 11, using Eqns. 4, 12 and 13, to provide

$$
\frac{w}{x_{d}}=\frac{3 F_{\max }}{2 \pi a^{2} p_{\max }}
$$

which relates a ratio of distances to a ratio of pressures. The maximum repulsive force $F_{\max }$, as given in Eqn. 1, is proportional to the particle radius $a$, while the maximum capillary force is inversely proportional to $a$. We therefore predict that the ratio $w / x_{d}$ is independent of particle radius, and only depends on the particle surface properties.

\section{DISCUSSION}

We have observed that the process of solidification of a directionally dried colloidal dispersion occurs in two stages. These have been argued to be an ordering of the colloidal particles into a non-touching rigid network, followed by the collapse of that network, and the irreversible aggregation of the particles. A simple theory was then developed, which balanced compressive capillary pressures against inter-particle electrostatic and van der Waals forces. This theory was able to accurately describe the experimental observations, and leads to predictions as to the scaling behavior of the two solidification fronts.

A multi-stage solidification process has previously been suggested in the drying of colloidal alumina $[7,26]$. In these studies, the height of a drying dispersion was monitored with a laser extensometer. The height levelled off as the dispersion solidified, but then underwent a steplike decrease of approximately $3 \%$ between the solidification and pore-emptying fronts [7, 27]. The interpretation given to this was that the particles were first held in some secondary minimum, before they were forced into intimate contact. This secondary minimum was ascribed to an additional, non-DLVO interaction [26]. In this work we have also observed a step-like change in film height, but show that it can be explained by a balance between DLVO forces, and the capillary force. We did not detect any other sharp changes in the film height, within the solidified film. 
In order to compare theory with experiment a number of simplifying assumptions were made, which require further discussion. The DLVO model is based upon pairwise additivity of potentials. This standard approach assumes that the interaction between each pair of particles is independent of the surrounding particles. For a concentrated array of particles this assumption becomes weak as overlapping electromagnetic fields cause a non-pairwise interaction. This contribution to the overall system energy is generally neglected, and it has been argued elsewhere [10] that it is small when $\kappa a \gg 1$. In our polystyrene latices, the minimum value of $\kappa a$ is 8 . For much smaller particles, the contribution of non-pairwise interactions may need to be considered.

The surface potential $\Phi$ was calculated in Eqn. 2, assuming a surface charge that was independent of the solvent salt concentration. For polystyrene latices prepared with potassium persulfate as an initiator, sulphate groups are integrated into the latex particles, leading to strong acidic surface groups [28]. Under these conditions a constant surface charge model is appropriate, and typically measured surface charge densities are between 0.2 and $1.5 \mu \mathrm{C} / \mathrm{cm}^{2}[11,19,29]$. In turn, in our $198 \mathrm{~nm}$ diameter polystyrene latices, Eqn. 2 predicts a surface potential of $-66 \mathrm{mV}$ at $1 \mathrm{mM} \mathrm{KCl}$ concentration. As the magnitues of $\Phi$ are in excess of $25 \mathrm{mV}$, the appropriate equations for the high-potential limit are used in Eqns. 1 and 2.

When modelling the capillary pressure, assumptions were introduced involving the solids volume fraction $\phi$, and its effect on the permeability $\kappa_{p}$ of the network. In evaluating Eqn. 11 it was assumed that the solidifying region was characterized by a representative value of $\phi=0.5$, while in deriving Eqns. 14 and 15 it was implicitly assumed that $\kappa_{p}$ was the same on both sides of the aggregation front. Relaxing these simplifications results in the scaling of the theoretical predictions by some constant. By varying the representative value of $\phi$ by \pm 0.1 , for example, the predicted curve in 6 (b) would shift up or down by a factor of 3 . If the permeability is allowed to change from $\kappa_{p}$ to $\kappa_{p}^{\prime}$ across the aggregation front, $x=w$, then the right-hand side of Eqns. 14 and 15 will be scaled by $\kappa_{p} / \kappa_{p}^{\prime}$. If the packing fraction in the aggregated region is in the range 0.64 to 0.74 , this factor is between 4 and 16 .

The collapse of an electrostatic barrier to aggregation has been noticed in centrifuge experiments with polystyrene dispersions similar to those we synthesized $[9,11]$. Dispersions were centrifuged until they formed solid pucks. These were then vigorously shaken in an attempt to re-disperse the solids. Below a certain height in each puck, the particles could not be re-dispersed. The pressure caused by the weight of the re-disperable layer under full acceleration was calculated, and equated with the critical aggregation pressure. Results were dependent on the particle surface chemistry, but the typical pressures required to effect aggregation were between 2 and $15 \mathrm{kPa}$. In most cases an increase from 1 to $10 \mathrm{mM} \mathrm{KCl}$ reduced the pressure required to induce aggregation by a factor of 2 to 4 . These results are in excellent agreement with the aggregation pressures that have been outlined here. This suggests that the corrections described in the preceding paragraph are small.

Our theory, up to the equilibrium water balance given in Eqn. 12, allows for a variable solidification front speed $v$. When $v$ increases, the pressure gradients in the film also increase. In particular, the pressure at the crack front, $x_{c}$, evaluated using Eqn. 7, depends on $v$. The simultaneous advance of many cracks, when the solidification fronts accelerate, can therefore be seen to result from a sudden increase in the magnitude of the capillary pressure at the crack tips. The intermittent behavior of the solidification fronts cannot be clearly attributed to any particular mechanism, but may be the result of fluctuations in the evaporation rate, or variations in the local strength of the particle network.

The self-assembly of colloidal particles into crystalline networks is the basis of the fabrication of photonic crystals (see e.g. [30]), and is a field where our results may be applied. For example, Juillerat et al. [31] studied surface, rather than directional, drying of silica particles, in order to understand the long-range ordering of photonic crystals. They suggested that a key aspect of colloidal self-assembly was that the particles pass through a stage allowing the rearrangement of particles within a crystal structure, before the dispersion fully solidifies. This stage corresponds to the region between the two solidification fronts identified in this paper.

Application of the simple methods presented here can also be made for estimating colloidal stability, or characterizing colloidal surface chemistry. Eqns. 11, 14 and 15 each provide a means of estimating the strength of the inter-particle interactions in a dispersion, in terms of visual observables, and process variables. These experiments can be performed by spreading a few hundred $\mu l$ of dispersion onto a slide, and observing the solidification region under an optical microscope. The pressureinduced transitions in dispersion structure can appear as changes in color or transparency of the film. By merely measuring the width of the transition region between the two solidification fronts provides, using Eqn. 14, an estimate of the maximum interaction force, $F_{\max }$, between a pair of particles. Alternatively, if the positions and velocities of the drying fronts are measured, Eqn. 11 gives $F_{\max }$. This method appears to be comparable in accuracy to the established method of measuring the critical aggregation pressure by centrifugation [9, 11], but requires considerably less dispersion per trial. It also offers advantages in the speed of evaluation, especially if the stability of multiple samples need to be compared.

\section{CONCLUSIONS}

Two distinct solidification fronts were seen during the directional drying of polystyrene and silica colloidal dispersions. These are visually apparent as changes in the 
color and transparency of the film. The region between these two fronts can support a rigid deformation, but is re-dispersed if flooded with water.

The leading solidification front was associated with the ordering of the particles, while the trailing front was associated with irreversible adhesion of particles. This interpretation is supported by a simple model, which shows that these behaviors arise naturally when the electrostatic and van der Waals forces, described by DLVO theory, are balanced against the compressive forces generated by fluid flow through a porous network. Using this model, a visual inspection of the solidification fronts is sufficient to estimate the maximum repulsive force between the dispersion particles.

\section{Acknowledgements}

The authors wish to thank M. O'Sullivan for assistance in synthesizing colloidal dispersions, and E. Eiser for helpful discussions. Funding from the Engineering and Physical Sciences Research Council [EP/E05949X/1] is gratefully acknowledged.

\section{Supplementary Information Provided}

Two supplemental movie figures are provided. Supplement 1 shows the dynamics of the ordering and aggregation fronts in a dispersion of $198 \mathrm{~nm}$ polystyrene. Supplement 2 shows the coupled irregular motion of the aggregation and fracture fronts, as seen in ludox AM. This information is available free of charge via the Internet at http://pubs.acs.org.
[1] R. D. Deegan, P. J. Petersan, M. Marder, and H. L. Swinney, Phys. Rev. Lett. 88, 014304 (2002).

[2] A. F. Routh and W. B. Russel, AIChE J. 44, 2088 (1998).

[3] R. C. Chiu, T. J. Garino, and M. J. Cima, J. Am. Ceram. Soc. 76, 2257 (1993)

[4] C. Allain and L. Limat, Phys. Rev. Lett. 74, 2981 (1995).

[5] E. R. Dufresne, E. I. Corwin, N. A. Greenblatt, J. Ashmore, D. Y. Wang, A. D. Dinsmore, J. X. Cheng, X. S. Xie, J. W. Hutchinson, and D. A. Weitz, Phys. Rev. Lett. 91, 224501 (2003).

[6] E. R. Dufresne, D. J. Stark, N. A. Greenblatt, J. X. Cheng, J. W. Hutchinson, L. Mahadevan, and D. A. Weitz, Langmuir 22, 7144 (2006).

[7] D. M. Holmes, R. V. Kumar, and W. J. Clegg, J. Am. Ceram. Soc. 89, 1908 (2006).

[8] L. Xu, S. Davies, A. B. Schofield, and D. A. Weitz, Phys. Rev. Lett. 101, 094502 (2008).

[9] A. E. J. Meijer, W. J. van Megen, and J. Lyklema, J. Colloid Interface Sci. 66, 99 (1978).

[10] C. N. Bensley and R. J. Hunter, J. Colloid Interface Sci. 92, 436 (1983).

[11] C. N. Bensley and R. J. Hunter, J. Colloid Interface Sci. 92, 448 (1983).

[12] N. Tsapis, E. R. Dufresne, S. S. Sinha, C. S. Riera, J. W. Hutchinson, L. Mahadevan, and D. A. Weitz, Phys. Rev. Lett. 94, 018302 (2005).

[13] J. B. Jones, J. V. Sanders, and E. R. Segnit, Nature 204, 990 (1964)

[14] J. V. Sanders, Acta Cryst. A24, 427 (1968).

[15] T. M. Truskett, S. Torquato, and P. G. Debenedetti, Phys. Rev. E 62, 993 (2000).

[16] P. N. Pusey and W. van Megen, Nature 320, 340 (1986).
[17] Y. Takeoka, M. Honda, T. Seki, M. Ishii, and H. Nakamura, ACS App. Mat. Interfaces 1, 982 (2009).

[18] R. H. Ottewill and J. N. Shaw, Kolloid Z Z Polym. 215, 161 (1967).

[19] J. Stone-Masui and A. Watillon, J. Colloid Interface Sci. 52, 479 (1975).

[20] W. B. Russel, D. A. Saville, and W. R. Schowalter, Colloidal dispersions (Cambridge University Press, Cambridge, 1989).

[21] I. D. Morrison and S. Ross, Colloidal Dispersions, Suspensions, Emulsions, and Foams (Wiley-Interscience, New York, 2002).

[22] R. J. Hunter and L. R. White, Foundations of colloid science (Clarendon Press, Oxford, 1987).

[23] F. A. Holland and R. Bragg, Fluid Flow for Chemical Engineers (Edward Arnold, 1995), 2nd ed.

[24] R. C. Chiu and M. J. Cima, J. Am. Ceram. Soc. 76, 2769 (1993).

[25] L. R. White, J. Colloid Interface Sci. 90, 536 (1982).

[26] D. M. Holmes, F. Tegeler, and W. J. Clegg, J. Euro. Ceram. Soc. 28, 1381 (2008).

[27] D. M. Holmes, Ph.D. thesis, University of Cambridge (2006).

[28] R. H. Ottewill and J. N. Shaw, Kolloid Z Z Polym. 218, 34 (1967).

[29] S. S. Madaeni and M. Ghanbarian, Polym. Int. 49, 1356 (2000).

[30] J. Zhang, Z. Sun, and B. Yang, Curr. Opin. Colloid In. 14, 103 (2009).

[31] F. Juillerat, P. Bowen, and H. Hofmann, Langmuir 22, 2249 (2006). 
ONLINE SUPPLEMENT 1: Motion of the ordering and aggregation fronts in a dispersion of $198 \mathrm{~nm}$ polystyrene particles, with $0.6 \mathrm{mM} \mathrm{KCl}$ added. A $1.1 \times 0.8 \mathrm{~mm}^{2}$ area is shown, at 30 times real speed, as viewed under a digital microscope. The opaque orange region of the film, in the upper section of the video, is liquid dispersion, where unordered particles randomly scatter light. The green central band contains particles that have crystallized, and are diffracting light in the visible spectrum, but which can be re-dispersed. The blue area at the bottom of the video contains particles that are irreversibly bound to each other. The two fronts advance in an intermittent way across the field of view, as the film dries.

ONLINE SUPPLEMENT 2: Magnified view of a $1.1 \times 0.8$ $\mathrm{mm}^{2}$ area of drying ludox $\mathrm{AM}$, in a $30 \mathrm{mM} \mathrm{KCl}$ solvent, played at 30 times real speed. The aggregation front is visible as a change in the film color, from dark grey to light grey. The ordering front cannot be clearly seen under these conditions. The velocity of the aggregation front is modulated, as was shown in $5(\mathrm{~b})$. When this front accelerates, there is a synchronous advance of the crack tips. The fringes behind the crack front show where the film de-bonds from the glass substrate. 